\title{
Measurement of Heart Rate using Photoplethsmography
}

\author{
Mehmet Merkepci ${ }^{1}$, M.Sadettin Ozyazici ${ }^{2}$ \\ ${ }^{1}$ Department of Electrical\&Electronics Engineering, University of Gaziantep, Gaziantep / Turkey \\ ${ }^{2}$ Department of Electrical\&Electronics Engineering Bahcesehir University, İstanbul / Turkey \\ ${ }^{1}$ merkepci@gantep.edu.tr*, ${ }^{2}$ sadettin.ozyazici@eng.bau.edu.tr
}

\begin{abstract}
Heart rate is a vital health parameter that is directly related to the soundness of the human cardiovascular system. This project describes a technique of measuring the heart rate through a fingertip using a PIC microcontroller. While the heart is beating, it is actually pumping blood throughout the body, and that makes the blood volume inside the finger artery to change too. This fluctuation of blood can be detected through an optical sensing mechanism placed around the fingertip. The signal can be amplified further for the microcontroller to count the rate of fluctuation, which is actually the heart rate.
\end{abstract}

Keywords: Microcontroller, heart rate, blood pressure, photoplethysmography

\section{Introduction}

Photoplethysmography (PPG) is a very effective technic to measure blood volume changes of tissue which has micro vascular bed [1]. PPG has common clinical and medical applications using technology in medical devices. To illustrate, pulse oximeter can be given an example. It can measure vascular diagnostics and digital beat-to-beat blood pressure measurement systems. PPG technology is consisting of two optic-optoelectronic devices/components. A light source and photo detector are needed. The light source is used to illuminate the skin and photo detector is used to evaluate the small variations in light intensity. The variations are related to changes in perfusion in the catchment volume. PPG is generally used non-invasively and operates at a red or a near infrared wavelength. The well-known waveform of PPG is the peripheral pulse, and it is synchronized with each heartbeat. In spite of its simplicity the roots of the different components of the PPG signal are not understood still completely. However, it is common accepted that PPG can provide valuable information about the cardiovascular system [2].

\section{Vital Signals}

In this section some information are given about heart rate and blood pressure which have very important information of human's health. Basically blood pressure (BP) and heart rate (HR) are different from each other. While your blood pressure is the force of your blood moving through your blood vessels, your heart rate is the number of times your heart beats per minute. They are two separate measurements and indicators of health. Heart rate and blood pressure do not necessarily increase at the same rate A rising heart rate does not cause your blood pressure to increase at the same rate. Even though your heart is beating more times a minute, healthy blood vessel get larger to allow more blood to flow through more easily. When you exercise, your heart speeds up so more blood can reach your muscles. It may be possible for your heart rate to double safely, while your blood pressure may respond by only increasing a modest amount [3]. 


\subsection{Heart Rate}

Heart rate (HR) is one of the most significant parameter among the vital signals that to measure for patient monitoring. The PPG pulse has both AC signal and DC signal. The AC signal component is related to the heart beat (HB) and so provides heart rate information. This information can be shown in PO. However, sometimes we can reduce confidence in the rate parameter because of a movement artefact or cardiac arrhythmia [4].

\subsection{Blood Pressure}

Blood Pressure (BP) which is generally known as arterial blood pressure [5] (ABP) is a very important indicator for human health and it is used to observe the physiological conditions of human in addition to other important signals like heart rate and blood oxygen saturation level. BP is classified in two categories. First is systolic blood pressure (SBP) and second is diastolic blood pressure (DBP).

DBP is the minimum pressure on walls of the arterial whereas SBP is the maximum peak pressure.

SBP and DBP may change during the day. Due to some factors such as stress, medicines, illness and exercises they can vary.

The human's BP is generally interpreted from the viewpoint of the SBP over DBP and it is determined in millimeters of mercury ( $\mathrm{mm} \mathrm{Hg}$ ). In generally, normal BP is approximately $80 / 120 \mathrm{mmHg}$ for the an adult. This value, however, changes depending on activity and medical condition by the nervous and other factors [6]. Low $\mathrm{BP}$ is known as hypotension and high is known as a hypertension [7]. They can range from moderate to severe, together with intense and chronic forms.

\section{Basically System Process}

As you seen before, the aim of the device is to measure heart rate which is actually above signal in figure 1.1 counted up the converted pulses in figure 1.2. The sensor consists of a super bright red LED and light detector (LDR). The LED needs to be super bright as the maximum light must pass spread in finger and detected by detector. When the heart pumps a pulse of blood through the blood vessels, the finger becomes slightly more opaque and so less light reached the detector. With each heart pulse the detector signal varies. This variation is converted to electrical pulse. This signal is amplified and triggered through an amplifier which outputs $+5 \mathrm{~V}$ logic level signal. Finally, the microcontroller determines the number of pulses between defined time interval and so heart rate of the person is obtained.

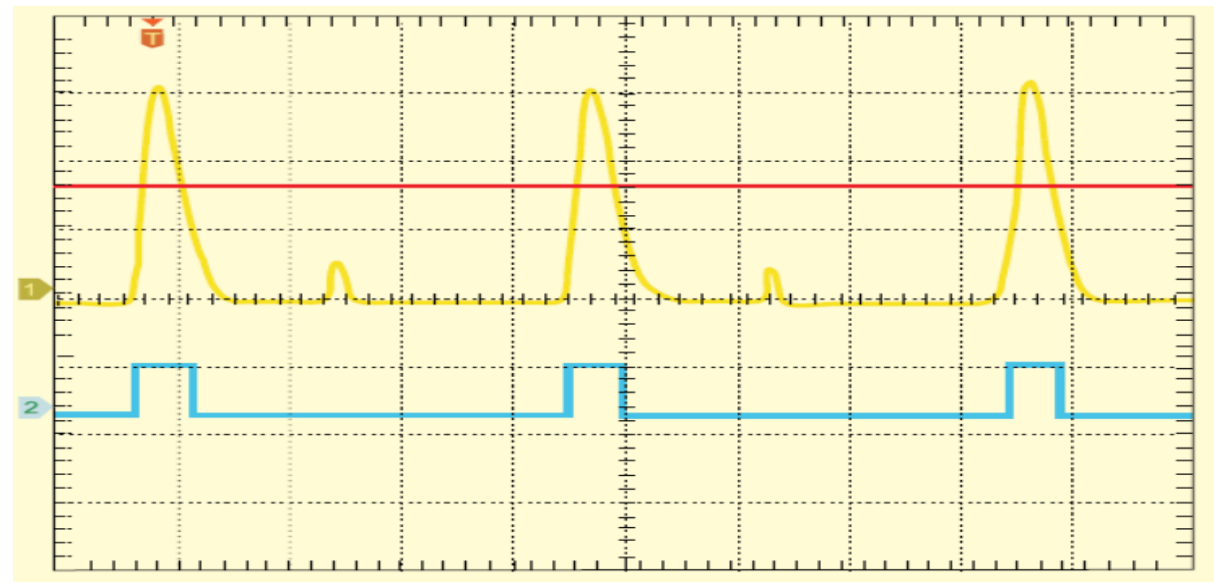

Fig. 1: The actual heart beat of human (1), the pulse demonstrations of heart beat (2).

In figure 2. block diagram of circuit's can be seen. 


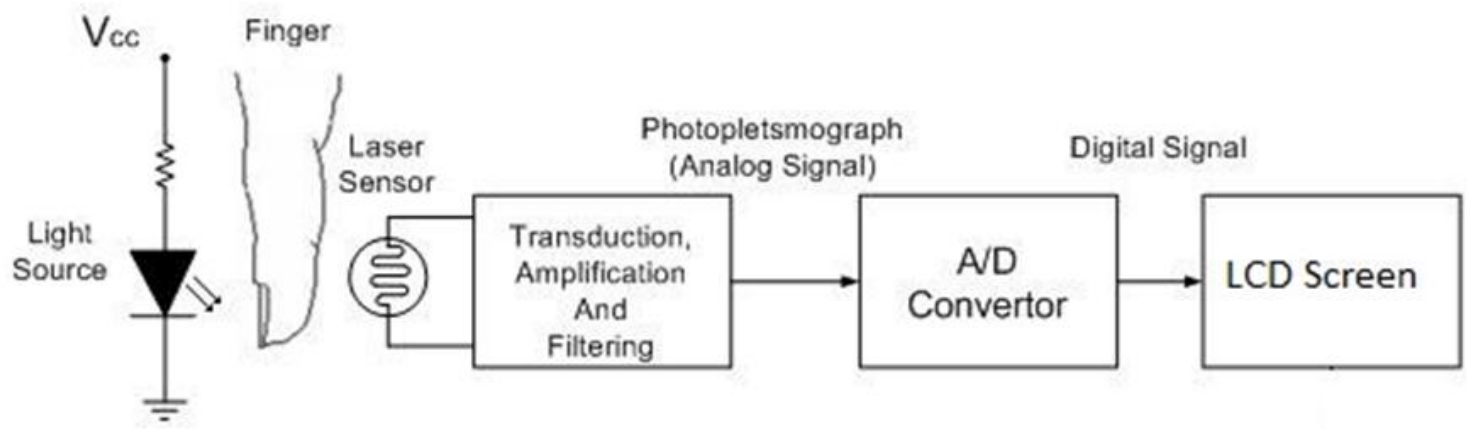

Fig. 2: Block diagram of circuit

A sample measurement can be seen in figure 3.

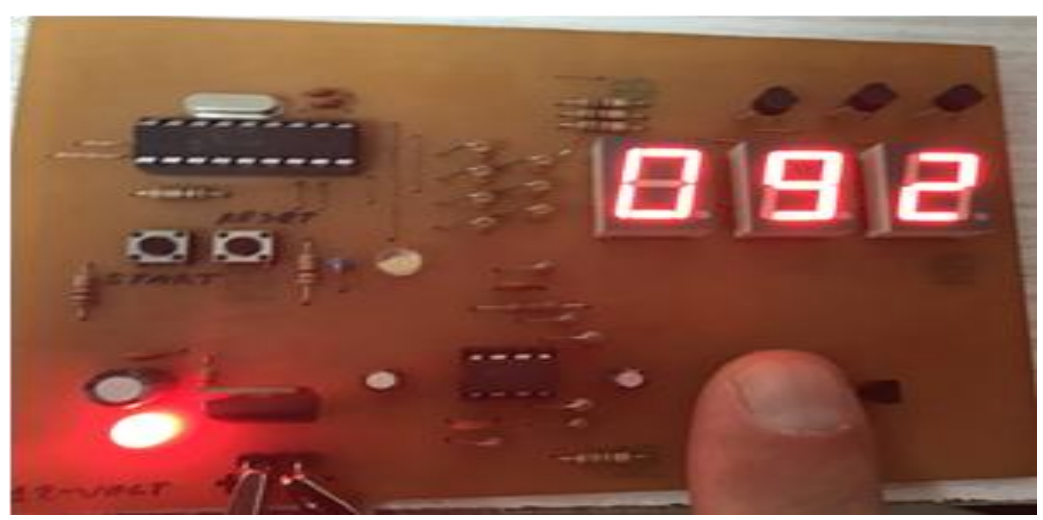

Fig. 3: Heart rate measurement

\section{Conclusion}

In this paper, a low-cost microcontroller based device is designed. The device has many advantages such as it can be used by nonprofessional people at home to measure heart rate. The measured values are compared with a standard PO device and very close values are obtained (see Table 1).

TABLE I: Experimental Values

\begin{tabular}{|c|c|c|}
\hline Persons & Our & Device \\
\hline 1 & 89 & 91 \\
\hline 2 & 94 & 95 \\
\hline 3 & 96 & 96 \\
\hline 4 & 98 & 99 \\
\hline 5 & 90 & 92 \\
\hline 6 & 94 & 93 \\
\hline 7 & 97 & 97 \\
\hline 8 & 88 & 89 \\
\hline 9 & 96 & 95 \\
\hline 10 & 100 & 101 \\
\hline
\end{tabular}

\section{References}

[1] Challoner, A. V. J. (1979) Photoelectric plethysmography for estimating cutaneous blood flow. In Non-invasive physiological measurements: 1.Rolfe, P. (Ed.), Academic Press, London, 125-151. 
[2] A.A.R. Kamal, J.B. Harness, G. Irving, A.J. Mearns, Skin photoplethysmography — a review, In Computer Methods and Programs in Biomedicine, Volume 28, Issue 4, 1989, Pages 257-269,

[3] http://www.heart.org/HEARTORG/Conditions/HighBloodPressure/GettheFactsAboutHighBloodPressure/BloodPressure-vs-Heart-Rate-Pulse_UCM_301804_Article.jsp\#.WehgTlt-odU

[4] Allen J, Photoplethysmography and its application in clinical physiological measurement Physiol. Meas. Vol.28 2007 R1-R39

[5] John G. Webster, Medical instrumentation, application and design, second edition, Houghton Mifflin Company, 1992

[6] Darovic, Gloriya Oblouk. Hemodynamic Monitoring: Invasive and Non-Invasive Clinical Application, 2nd edition. Philadelphia: W.B. Saunders Company,1995.

[7] U.S. Department Of Health And Human Services National Institutes of Health National Heart, Lung, and Blood Institute National High Blood Pressure Education Program NIH Publication No. 03-5232 May 2003 